\title{
Experimental Demonstration of All-Optical Regeneration Using an MMI-SOA
}

Jan De Merlier, Student Member, IEEE, Geert Morthier, Senior Member, IEEE, Steven Verstuyft, Tom Van Caenegem, Student Member, IEEE, Ingrid Moerman, Member, IEEE, Peter Van Daele, Member, IEEE, and Roel Baets, Senior Member, IEEE

\begin{abstract}
A novel all-optical $2 \mathrm{R}$ regenerator based on a multimode interference coupler (MMI) semiconductor optical amplifier is presented. Static measurements of the transfer function reveal a digital transfer characteristic and a high increase in extinction ratio. The experiments are in good agreement with simulations, which have been done using a modified beam propagation method program. The device has a high tolerance to the MMI length. It has been fabricated in an all-active layout, avoiding the need for active/passive integration and is very compact.
\end{abstract}

Index Terms-All optical, MMI, regeneration, semiconductor optical amplifier.

\section{INTRODUCTION}

D UE TO THE accumulation of noise introduced by the amplifiers, jitter and dispersion, the data signals are seriously degraded while propagating in an optical network. To avoid the electronic bottleneck, the integration of all-optical regenerators will be required in future high-speed wavelength-division-multiplexing (WDM) systems to assure a sufficient network node cascadability. Most of the regenerators, proposed so far, perform regeneration with simultaneous wavelength conversion which makes them complicated devices needing a continuous-wave (CW) laser and a filter at the output [1], [2]. 2R-regeneration making only use of the input signal has been demonstrated using a Michelson interferometer. This type of regenerator has been operated at $40 \mathrm{~Gb} / \mathrm{s}$ [3]. However, the Mach-Zehnder interferometer (MZI), as well as the Michelson interferometer have the disadvantage that they lead to rather large chip sizes. In addition, to get a good regeneration characteristic, a cascade of several MZIs is needed [4]. Some time ago, we have proposed another type of $2 \mathrm{R}$ regenerator based on a multimode interference coupler (MMI)-semiconductor optical amplifier (SOA) with the potential of a digital static regeneration characteristic and a large increase of extinction ratio [5]. In this letter, the simulation of this device is described and first experimental results are shown.

\section{CONCEPT}

The MMI consists of a broad multimodal waveguide with inand output waveguides to couple the light in and out. When a step-index profile is assumed the following relationship is ob-

Manuscript received August 03, 2001; revised January 10, 2002. The work was supported by the Institute for the Promotion of Innovation by Science and Technology in Flanders (IWT).

The authors are with the Department of Information Technology, Ghent University, Ghent, Belgium (e-mail: jan.demerlier@intec.rug.ac.be).

Publisher Item Identifier S 1041-1135(02)03251-2.

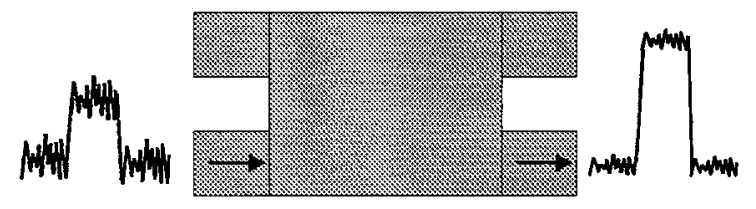

Fig. 1. Schematic of MMI-SOA based regenerator.

tained between the different propagation constants of the modes in the broad waveguide [6]:

$$
\beta_{0}-\beta_{\nu} \simeq \frac{\pi}{3 L_{\pi}} \nu(\nu+2) \text { with } L_{\pi}=\frac{4 n_{r} W_{\mathrm{eff}}^{2}}{3 \lambda}
$$

with $\beta_{\nu}$ the propagation constant of the $\nu_{\text {th }}$ order mode. The length $L_{\pi}$ corresponds to the beat length of the two lowest order modes with $n_{r}$ the effective index of the ridge and $W_{\text {eff }}$ the effective width of the waveguide including the penetration of the modes in the neighbor material. $\lambda$ is the wavelength in vacuum. From expression (1) it is possible to derive some self-imaging properties of the input field at certain distances in the waveguide. In case of a $2 \times 2 \mathrm{MMI}$ as shown in Fig. 1, at a distance of $3 L_{\pi}$ the excited modes interfere such that the input field is coupled to the output waveguide for the cross state. When this component is fabricated in an SOA-layer structure, nonlinear effects influence the behavior of the device. At low input powers, the device works in the linear regime and all the light is coupled to the output waveguide for the cross state. When the input power is increased saturation sets in and causes the step-index profile to be disturbed due to the influence of the lateral power distribution on the electron density, which in turn has its effect on the real and imaginary part of the refractive index

$$
n(x)=n_{r}+\Gamma\left(\beta N(x)+i \frac{a}{2 k_{0}}\left(N(x)-N_{0}\right)\right)
$$

with $n_{r}$ the effective refractive index of the ridge, $\Gamma$ the confinement factor, $\beta$ the antiguiding factor, $N(x)$ the electron density, $a$ the differential gain, $k_{0}$ the wave vector, and $N_{0}$ the transparency electron density. This implies that the propagation constants of the different modes in the broad waveguide are changed in a mode-dependent way and eventually will interfere more constructively at the output waveguide for the bar state. Based on this effect regeneration can be obtained.

\section{Simulation Results}

The simulations have been obtained using a two-dimensional static beam propagation method, linked to an external program 


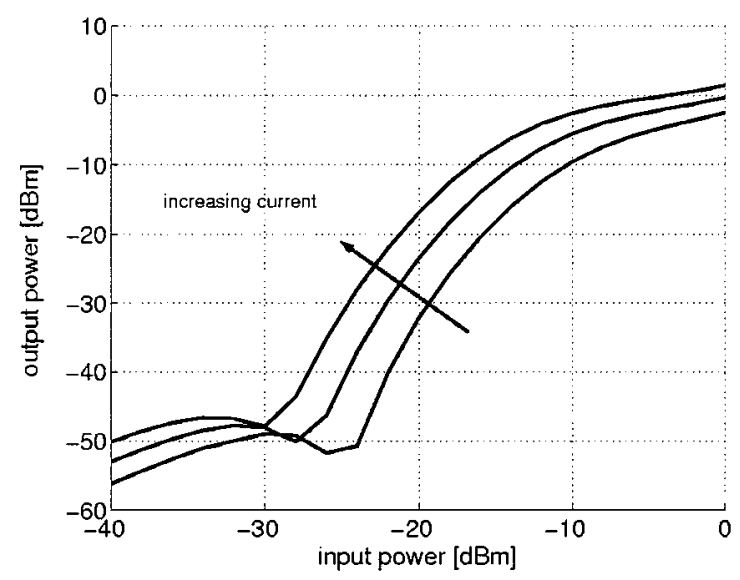

Fig. 2. Simulations of regeneration curve at different currents.

which takes into account the interaction between the carriers and the light. The unimolecular, bimolecular and Auger recombination mechanisms have been considered and the gain is assumed to be linearly dependent on the electron density. The lateral electron density profile is calculated by solving the diffusion equation after each propagation step of $5 \mu \mathrm{m}$.

$$
\frac{J}{q d}-D_{\mathrm{eff}} \frac{\partial^{2} N}{\partial x^{2}}-A N-B N^{2}-C N^{3}-\frac{\Gamma a\left(N-N_{0}\right) P(x)}{\hbar \omega b d}=0 .
$$

With $J$ the current density, $q$ the elementary charge, $d$ the thickness of the active layer, $D_{\text {eff }}$ the effective diffusion coefficient, $A, B$ and $C$ the different recombination parameters, $\hbar$ the reduced Planck constant, $\omega$ the optical angular frequency and $b$ the width of the waveguide. As mentioned before, the working principle of the MMI is based on the step index-profile. Because lateral carrier diffusion in the active layer of a shallowly etched ridge SOA-waveguide causes a nonhomogeneous carrier distribution under the ridge, the lateral refractive index profile is already disturbed in the linear regime and has an influence on the self-imaging properties of the MMI-SOA. Therefore, the ridge waveguide has to be etched through the active layer. This causes a homogeneous distribution of the carriers under the ridge when surface recombination is negligible.

Fig. 2 shows calculated transfer functions at current densities of 3000,3500 , and $4000 \mathrm{~A} / \mathrm{cm}^{2}$. It gives the output power in the fundamental mode in the bar state output waveguide as a function of the input power in the fundamental mode. The MMI has a width of $8 \mu \mathrm{m}$. The input and output waveguides have a width of $3 \mu \mathrm{m}$. The simulation results give proof of a digital transfer characteristic of which the decision point can be swept by changing the current.

\section{FABRICATION AND CHARACTERISTICS}

MMI-SOAs have been fabricated with a width of $8 \mu \mathrm{m}$. The width of the in- and output waveguide are $3 \mu \mathrm{m}$. This makes it a tolerant device with respect to the width of the device. The layer structure consists of eight 8-nm-thick $1 \%$ compressively strained InGaAsP quantum wells (QWs) with InGaAsP (Q1.25) barriers of thickness $15 \mathrm{~nm}$. This layer stack has been sandwiched between two InGaAsP (Q1.25) layers of $90 \mathrm{~nm}$. The layer structure has been grown using metal-organic vapor phase

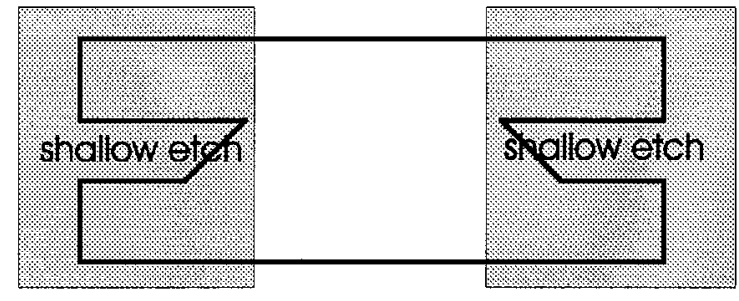

Fig. 3. Detail of the MMI-facet.

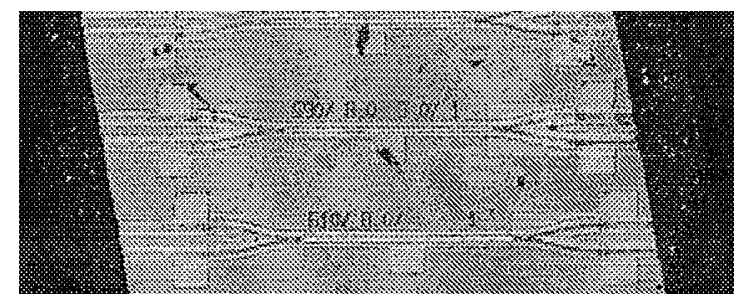

Fig. 4. Photograph of MMI-SOA chip.

epitaxy (MOVPE). A combination of wet etching and reactive ion etching (RIE) has been used to define the ridges of the ridge-type waveguides. The ends of the MMI facets have been tilted at $45^{\circ}$ to avoid reflections at the MMI-facets. The in- and output waveguides have been etched shallowly and the MMI itself has been etched deeply to avoid the current spreading. The transition from deep etching to shallow etching has been performed just inside the MMI, as shown in Fig. 3, to minimize the reflection at the MMI-facets. The output waveguides have been tilted at an angle of $10^{\circ}$, no AR-coating has been applied. The device has been fabricated in an all-active layout. To avoid saturation effects in the in- and output waveguides they have been provided with separate electrodes which are biased near transparency. The total size of the device, as in Fig. 4, is only 1500 $\mu \mathrm{m}$ including the peripheral waveguides.

\section{Measurement Results}

The light has been coupled into the chip using a lensed fiber. No tapers were used and no antireflection coating has been deposited on the chip facet, causing a coupling loss of about 8 $\mathrm{dB}$. The output coupling has been realized by using an objective lens, then the light is coupled into a single-mode fiber using a GRIN-lens. The output is sent to a spectrum analyzer to measure the power at the wavelength of the input signal in a bandwidth of $0.1 \mathrm{~nm}$. Due to the strong polarization dependence of the gain in compressively strained QWs, the polarization of the input light had to be controlled. Fig. 5 shows the output power in the bar state output waveguide as a function of the input power of an MMI with a length of $570 \mu \mathrm{m}$ for various currents at a signal wavelength of $1555 \mathrm{~nm}$. The measurements correspond very well to the simulations and show a flat response at high input power. The low detected output powers are due to the poor coupling efficiency of the light from the chip into the single-mode fiber after the objective lens and the graded index (GRIN) lens. The flat high power side will allow an increase in signal to noise ratio, while the steep transition between the low and high power side will allow an increase in extinction ratio. Extinction ratio improvements of more than $8 \mathrm{~dB}$ are obtained. Fig. 6 presents 


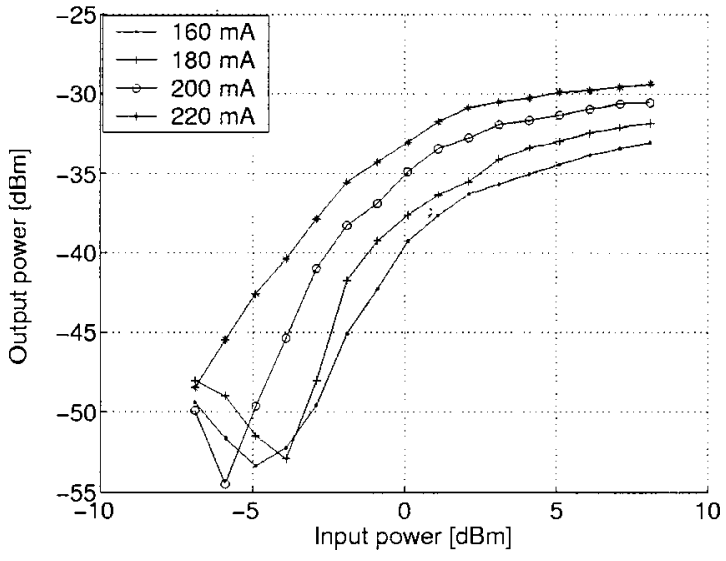

Fig. 5. Static transfer function of MMI with length $570 \mu \mathrm{m}$ at different currents.

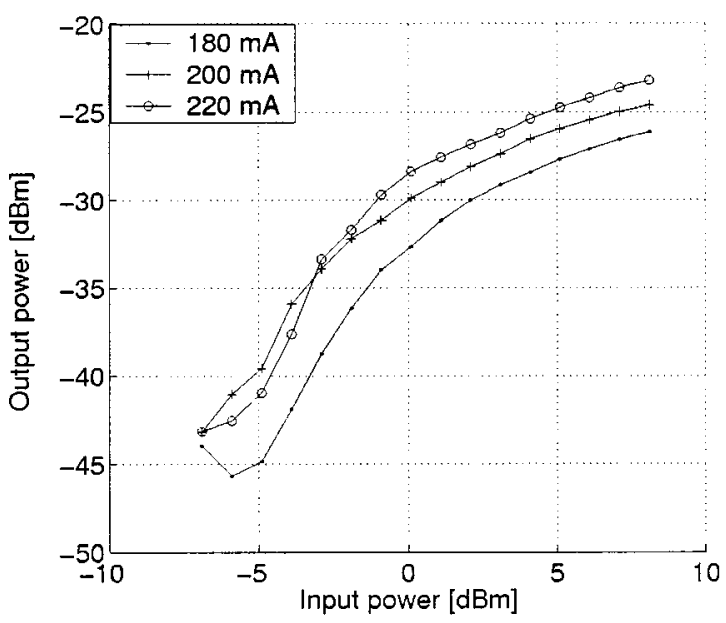

Fig. 6. Static transfer function of MMI with length $550 \mu \mathrm{m}$ at different currents.

the measurements of an MMI with a length of $550 \mu \mathrm{m}$ at different currents, still obtaining good results. Experimental results on an MMI of $580 \mu \mathrm{m}$ in length have been presented in [5]. This means that the MMI-SOA performs good regenerative properties over a range of $30 \mu \mathrm{m}$. Because the length of the MMI is related to the width, following the expression in (1), this tolerance in length corresponds to a tolerance in width of $\pm 0.1 \mu \mathrm{m}$ which is technologically acceptable.

\section{CONCLUSION}

A $2 \mathrm{R}$ regenerator is presented based on a 2 by 2 MMI-SOA. Simulations of the device, obtained using a modified two-dimensional BPM program, have been shown. The device has been fabricated and tested. The measured regeneration characteristics agree well with the simulations and show a digital form and offer an extinction ratio improvement of about $8 \mathrm{~dB}$. The component shows a regenerative behavior with a MMI-length tolerance of $30 \mu \mathrm{m}$, corresponding to a width tolerance of 0.1 $\mu \mathrm{m}$. The dynamics of the device has not been treated in this letter but is subject to investigation and is expected to be similar to the dynamics of single moded SOAs.

\section{ACKNOWLEDGMENT}

The authors would like to thank H. Sergeant and L. Haentjens for technical support.

\section{REFERENCES}

[1] D. Wolfson, A. Kloch, T. Fjelde, C. Janz, B. Dagens, and M. Renaud, "40 Gb/s all-optical wavelength conversion, regeneration and demultiplexing in an SOA-based all-active Mach-Zehnder interferometer," IEEE Photon. Technol. Lett, vol. 12, pp. 332-334, Mar. 2000.

[2] O. Brox, A. Kilk, C. Caspar, D. Hoffmann, M. Möhrle, G. Sahin, and B. Sartorius, "Q-switched laser module for regenerative wavelength conversion," in Proc. ECOC 2000, vol. 2, pp. 57-58.

[3] D. Wolfson, P. Hansen, A. Kloch, T. Fjelde, C. Janz, A. Coquelin, I. Guillemot, F. Gaborit, F. Poingt, and M. Renaud, "All-optical 2R regeneration at $40 \mathrm{Gbit} / \mathrm{s}$ in an SOA-based Mach-Zehnder interferometer," in Tech. Dig. OFC, San Diego, CA, 1999, Postdeadline Paper 36.

[4] B. Lavigne, D. Chiaroni, P. Guerber, L. Hamon, and A. Jourdan, "Improvement of regeneration capabilities in semiconductor optical amplifier-based 3R regenerator," in Tech. Dig. OFC, San Diego, CA, 1999, Paper TuJ3

[5] J. De Merlier, G. Morthier, T. Van Caenegem, R. Baets, I. Moerman, and P. Van Daele, "Experimental demonstration of $15 \mathrm{~dB}$ extinction ratio improvement in a new $2 \mathrm{R}$ optical regenerator based on an MMI-SOA," in Proc. ECOC, Amsterdam, The Netherlands, 2001, Paper Th.F.2.4.

[6] L. B. Soldano and E. C. M. Pennings, "Optical multi-mode interference devices based on self-imaging: Principles and applications," J. Lightwave Technol., vol. 13, pp. 615-627, Apr. 1995. 